\title{
Sexualidades, Política e Estado na América Latina: elementos críticos a partir de um debate Sul-Sul
}

Sexualidades, Política y Estado en América Latina: elementos críticos a partir de un diálogo Sur-Sur

Sexuality, Politics and State in Latin America: critical elements based on a South-South dialogue

\author{
Mario Pecheny \\ Universidad de Buenos Aires, Buenos Aires, Argentina.
}

\section{Rafael de la Dehesa}

City University of New York, New York, Estados Unidos.

\begin{abstract}
Resumo
O artigo "Sexualidades, Política e Estado na América Latina: elementos críticos a partir de um debate Sul-Sul" discute, a partir das contribuições de dois diálogos (um Latino Americano e outro Sul-Sul), algumas tendências políticas e intelectuais sobre direitos sexuais e política na América Latina, com o objetivo de mostrar certas tensões e perplexidades dos avanços legislativos e políticos em meio a crises e desigualdades persistentes.
\end{abstract}

Palavras-chave: Direitos Sexuais, Sexualidade, Política, América Latina.

\section{Resumen}

El texto "Sexualidades, política y Estado en América Latina: elementos críticos a partir de un diálogo Sur-Sur" discute, sobre la base de las contribuciones a dos Diálogos (uno Latinoamericano, y uno Sur-Sur), algunas tendencias políticas e intelectuales sobre derechos sexuales y política en América Latina, con el fin de mostrar ciertas tensiones y perplejidades de avances legislativos y políticos en medio de crisis y desigualdades persistentes.

Palabras Clave: Derechos sexuales, Sexualidad, Política, América Latina.

\begin{abstract}
Based on contributions to two Dialogues (one Latin American, one South-South), the text "Sexualities, politics and State in Latin America: critical elements from a South-South Dialogue" discusses some political and intellectual trends related to sexual rights and politics
\end{abstract}


in Latin America. The objective is to show some tensions and paradoxes raised by political and legal advances amidst persistent social crises and inequalities.

Keywords: Sexual rights, Sexuality, Politics, Latin America.

El continente latinoamericano corresponde al extremo Occidente del mundo, según la expresión de Alain Rouquié (1987), pero políticamente la región se considera “del Sur". A pesar de la evidente heterogeneidad y persistentes desigualdades, una historia común de luchas y esperanzas autorizan a hablar de América Latina como una unidad, una región que es capaz de albergar al mismo tiempo violentos crímenes contra lesbianas, trans y gays, y la mayor reunión política por los derechos LGBT del mundo (la marcha del orgullo en San Pablo), el matrimonio igualitario o una ley avanzada de identidad de género (ambos en Argentina); una región que tiene una larga tradición feminista, y las mayores y arbitrarias restricciones respecto del aborto.

En agosto de 2009, en Río de Janeiro tuvo lugar un Diálogo Latinoamericano sobre Sexualidad y Geopolítica, alentado y organizado por Sexuality Policy Watch (ver cada una de las contribuciones en Corrêa \& Parker, 2011, en cuyos aportes abreva este texto), y un nuevo encuentro tuvo lugar en la misma ciudad en septiembre de 2011, esta vez a nivel interregional. El texto que sigue sintetiza alguno de los puntos que hemos presentado en ese segundo encuentro, con el gran estímulo intelectual de Sonia Corrêa. Esa presentación tenía un doble objetivo: (a) retomar los núcleos críticos levantados en el diálogo latinoamericano de 2009, y (b) hacerlo de un modo que fuera comprensible para quienes no pertenecen a la región latinoamericana. Apuntando a relevar las realidades políticas que confrontan los movimientos por derechos sexuales en la región, las discusiones se organizaron en cuatro áreas: 1) posibilidades y límites del compromiso con el Estado y las sociedades políticas; 2) articulaciones entre militancia $y$ comunidad científica; 3) papel político de las religiones organizadas; 4) impacto de las transformaciones económicas en el escenario socio-sexual. Las presentaciones y gran parte de las discusiones del Diálogo Latinoamericano se encuentran publicadas (Correa \& Parker, 2011); a partir de ellas hemos organizado el presente artículo, con énfasis en la primera área mencionada.

En la región, en esta última ola de democratización, los progresos en el reconocimiento formal de derechos sexuales, reproductivos y de género son significativos. Cambios en las leyes de 
familia establecieron relaciones más igualitarias entre varones y mujeres dentro del matrimonio, eliminaron las distinciones legales entre hijos nacidos dentro o fuera de un matrimonio, y legalizaron el divorcio (el último país, Chile, lo hizo en 2004). En varios países, como Brasil, Colombia y Uruguay, se avanzó hacia maneras más amplias de comprender la familia a través del reconocimiento de parejas del mismo sexo. En 2009, el casamiento entre personas del mismo sexo fue reconocido en la Ciudad de México y en 2010, con amplio apoyo popular, en la Argentina (Clérico \& Aldao, 2010). La legislación antidiscriminatoria ocupa un lugar central en los cuerpos legales de toda la región. En 1998, Ecuador fue el segundo país en el mundo (el primer país fue Sudáfrica) en instituir protecciones constitucionales contra la discriminación basada en la orientación sexual y, en 2008, el primero en la región en contemplar también la identidad de género. A nivel regional, la Organización de Estados Americanos adoptó la Convención Interamericana para la Prevención, Castigo y Erradicación de la Violencia contra la Mujer (Convención de Belém do Pará) en 1994, y en 2008 aprobó por unanimidad una resolución condenando las violaciones de derechos humanos motivadas por la orientación sexual e identidad de género. Esto sumado a la campaña en curso de activistas por la adopción de una Convención Interamericana de Derechos Sexuales y Reproductivos habla de un cambio más amplio en términos de la política sexual y de género tanto como de la importancia creciente de su dimensión trasnacional. En 2012, Argentina aprobó una ley de Identidad de Género pionera en el mundo.

Sin embargo, estos logros no han sido parejos, tanto en lo que refiere a diferencias en el reconocimiento formal de los derechos sexuales según los países y áreas de políticas, como en términos de su impacto social más amplio, aun donde los derechos están reconocidos formalmente (Pecheny \& de la Dehesa, 2010, 2011). En verdad, la propia noción de "América Latina" plantea dilemas para el análisis (Corrêa \& Parker, 2011:), con el riesgo de oscurecer panoramas sexuales y sociales muy diferentes entre y dentro de las naciones que integran la región. La heterogeneidad en la organización social y los sentidos atribuidos al deseo sexual, el cuerpo, género y reproducción se localiza particularmente a través de clivajes y fronteras de clase, étnicas, raciales $y$ regionales.

Tales diferencias y desigualdades plantean cuestiones sobre las limitaciones de las políticas sexuales llevadas a cabo a través de las estructuras formales de las esferas públicas nacionales o internacionales, del lenguaje de los 
derechos, e incluso de modo más amplio sobre los límites de las democracias políticas emergentes. Las páginas que siguen no buscan ofrecer un relevamiento exhaustivo de las políticas sexuales en la región sino subrayar algunas tendencias significativas identificadas en el mencionado Diálogo y que pueden proveer una base para discusiones.

\section{Política, sexualidad y Estado en América}

\section{Latina}

Al pensar en sexualidad y política en América Latina, estamos hablando de sociedades y regímenes políticos centrados en el Estado, es decir en los que el Estado ha jugado históricamente un papel fundamental en la constitución de las reglas y la definición de los actores sociales y políticos. En este sentido, por ejemplo, si se usa la categoría analítica de gubernamentalidad (gouvernementalité), retomada de Michel Foucault (Foucault, 2004; Brown, 2006; Lascoumes \& Le Galès, 2007) ${ }^{1}$, debe ser entendida pues como una gubernamentalidad "centrada en el Estado". Pero el Estado latinoamericano no es una entidad monolítica, sino una "cacofónica" o incluso "esquizofrénica". Es el mismo Estado, con todas sus caras y manos, el que garantiza el estado de derecho y los derechos humanos, y el responsable (por no proteger o por ser el ejecutor directo) de la violación de derechos y el no reconocimiento de sujetos y prácticas sexuales, incluso de perpetrar asesinatos y ejercer directamente la violencia (a las mujeres, a las personas LGBT, etc.).

Dos paradojas centrales enmarcan en este contexto la comprensión crítica de los esfuerzos del activismo en derechos sexuales por involucrar al Estado y la sociedad política. La primera concierne el momento histórico contradictorio en el cual se consolidaron los movimientos sociales en materia de política sexual. $\mathrm{Si}$ bien las transiciones desde los autoritarismos a gobiernos democráticos a lo largo de casi toda la región, han abierto en diverso grado espacios para el diálogo, prometiendo políticas más inclusivas, éstas coincidieron con la crisis de la deuda en los años 1980 y la subsecuente adopción de políticas neoliberales de ajuste estructural, que magnificaron la polarización económica y reforzaron todas las formas de exclusión social. La segunda paradoja, más general, se refiere a la dificultad intrínseca de traducir el campo del erotismo, que implica una cierta fluidez en las identidades y los deseos, en los lenguajes de la legislación y las políticas públicas, un proceso que necesariamente implica volver rígidas las categorías sociales y reificar las identidades, contribuyendo a su vez a la competencia 
entre movimientos sociales y a formas de exclusión a menudo no reconocidas (Pecheny, 2010). Es en este marco paradojal que, luego de presentar un panorama rápido sobre la articulación del género y la sexualidad con la historia de la formación estatal y las principales expresiones del activismo y movimientos sociales contemporáneos sobre derechos sexuales en la región, examinaremos críticamente algunas limitaciones en este proyecto.

\section{Sexualidad y representación política:} ayer y hoy

Las repúblicas latinoamericanas que emergieron de las guerras de independencia del siglo XIX heredaron un orden social profundamente estratificado según clivajes de raza, género y clase social. El privilegio sexual, político y económico masculino estaba sancionado y protegido legalmente. Las élites políticas consideraban a la familia patriarcal como la base de una sociedad estable y una nación bien ordenada. Las leyes arraigadas en las tradiciones legales ibéricas y el derecho canónico instituyeron dicho patrón, cristalizando la autoridad del marido sobre la persona y la propiedad de la mujer (potestad marital) y la autoridad del padre sobre los hijos (patria potestad). Se establecieron así distinciones entre las mujeres casadas y no casadas, y al interior de las mujeres no casadas, y entre mayores y menores de edad. Rígidas restricciones limitaban la autoridad legal de las mujeres casadas sobre los hijos y su capacidad jurídica para firmar contratos, iniciar juicios y controlar la propiedad. Las viudas recobraban el control de su propiedad mientras no se volvieran a casar, y tanto ellas como las mujeres solteras por encima de la mayoría de edad podían contratar y ejercer determinados derechos civiles aunque no políticos - que disfrutaban los varones. Varones y mujeres eran asimismo juzgados de manera diferente bajo la ley criminal, particularmente en el área de los delitos contra el honor, que reforzaban las prerrogativas masculinas y las normas sociales restrictivas relativas a la virtud de la mujer. En parte por la débil capacidad estatal y la confianza de las élites en la “estabilidad patriarcal", las primeras reformas en el derecho de familia se produjeron bastante tiempo después de los procesos de independencia (Dore, 2000: 19; Petracci \& Pecheny, 2007). Los proyectos modernizadores de las élites liberales y, luego, positivistas comenzaron un paulatino proceso de secularización que asumió formas diversas según los países. Estudios históricos críticos recientes desafiaron los puntos de vista teleológicos que consideran las reformas liberales como pasos en el recorrido progresista hacia la 
emancipación femenina, y subrayan por el contrario sus efectos contradictorios en las relaciones de género, su impacto variable en diferentes grupos de mujeres, y sus concurrentes articulaciones con crecientes tecnologías de control social en medicina, salud pública, protección familiar, entre otras. Por ejemplo, mientras que algunas mujeres se beneficiaron por la institución del matrimonio civil y los pasos hacia la legalización del divorcio (yendo más lejos en México y las repúblicas centroamericanas que en los países de América del Sur), las reformas que terminaron con la herencia equitativa obligatoria, guiadas por la creencia en que preservar la unidad del patrimonio privado facilitaría la marcha del progreso, perjudicaron una institución que garantizaba a algunas mujeres compartir su propiedad familiar y una porción de autonomía (Deere \& León, 2001). De modo similar, mientras que la sodomía se despenaliza en gran parte de la región como reflejo de las reformas legales francesas y su modelo del Código Civil, otras leyes que regulaban la moral y buenas costumbres, la corrupción de menores, los atentados a la decencia pública, y la vagancia, permitieron la continua represión policial para mantener los órdenes raciales, sexuales y de género. Informadas por supuestas teorías de racismo científico y temores ante la degeneración nacional, las élites liberales priorizaron proyectos de modernización por sobre los ideales igualitarios $\mathrm{y}$ de libertades individuales, estableciendo repúblicas oligárquicas altamente excluyentes. En ese marco se explican los esfuerzos de las élites por transformar las poblaciones nacionales mediante el fomento de la inmigración europea, y el desplazamiento forzado de las comunidades indígenas a través de medidas que iban desde las campañas genocidas en el Cono Sur hasta la privatización de las tierras comunales en México, así como los esfuerzos tempranos por regular la prostitución y controlar las enfermedades venéreas.

Las repúblicas oligárquicas excluyentes abrieron paso a regímenes populistas inclusivos, que tuvieron un papel transformador en toda la región hasta bien entrado el siglo XX. Algunos con aspiraciones revolucionarias, otros con ambiciones más conservadoras atravesando todo el espectro político -, los líderes populistas adoptaron las primeras leyes laborales importantes y políticas de bienestar social, al tiempo que reorientaron las economías de enclave exportador de materias primas hacia modelos de industrialización por sustitución de importaciones y hacia los mercados internos. Siendo las primeras experiencias de movilizaciones de masas, los gobiernos 
populistas apuntaron a incorporar las crecientes clases medias urbanas y sectores populares, estableciendo vías corporativas de representación que asociaron (y subordinaron) a las organizaciones obreras a partidos políticos $y / o$ directamente al Estado.

Históricamente, las mujeres de la región habían estado involucradas en actividades de beneficencia, consideradas una extensión natural de los rasgos naturalmente femeninos. Con la expansión de la regulación estatal a nuevas y más íntimas esferas de las relaciones sociales, las mujeres, particularmente aquellas de las clases más altas, habían comenzado a participar de las políticas de bienestar social y de los movimientos eugenésicos, intentando "llevar la familia a la mirada de los reformistas" (Molyneux, 2000; Stepan, 1991). Las activistas de la primera ola feminista en América Latina, muchas de ellas vinculadas a movimientos socialistas y anarquistas, también alcanzaron sus más tempranos éxitos políticos. Movilizándose de manera similar a los modos corporativos, las mujeres en México organizaron Ligas Feministas, que superaron las 55.000 afiliadas en los años 1920, estableciendo fuertes lazos con el liderazgo post-revolucionario. Reflejando una orientación trasnacional aún hoy significativa, en la Ciudad de México se realizó en 1923 una Primera Conferencia
Feminista de la Liga Panamericana de Mujeres para discutir temas como acceso al trabajo, el derecho al sufragio $y$ planificación familiar, y demandaron un "único estándar moral respecto de los asuntos sexuales para varones y mujeres" (Cano, 1987: 26; Lau Jaivén, 2009).

En los procesos populistas, las mujeres también participaron de los procesos inclusivos, en un doble movimiento que las incorpora y reproduce el lugar socialmente esperable para ellas según la división jerárquica de género.En Argentina, el Partido Peronista Femenino, fundado en 1949, llegó a congregar en tres años a medio millón de mujeres (Cano, 1987) y habilitando una inédita participación de mujeres en el congreso. Hacia finales de los años 1950, a lo largo de América Latina estaba reconocido el sufragio femenino, al tiempo que un número creciente de mujeres entraban al mercado de trabajo y a las universidades.

Las décadas de 1960 y 1970 significaron una vuelta atrás de los populismos en muchas partes de la región, cuando dictaduras y gobiernos autoritarios apuntaron a reinstalar el orden social jerárquico desafiado por variados actores sociales, con períodos de sangrienta represión que diezmó gran parte de los movimientos sociales, populares y de izquierda. Las transiciones desde el autoritarismo a las democracias políticas 
que comenzaron a fines de los años 1970 y continuaron hasta los años 1990, crearon el escenario para los contemporáneos movimientos sociales de derechos sexuales, que emergieron en el seno de movimientos más amplios por cambios democráticos y aun revolucionarios. En verdad, gran número de las primeras militantes feministas y lesbianas, y los primeros militantes gays, tenían una historia de militancia en partidos socialistas, grupos estudiantiles radicalizados $\mathrm{y}$ organizaciones revolucionarias. Al encontrarse con una cultura política heterosexista y masculinista, y no sin grandes dilemas biográficos, se volvieron hacia los movimientos sociales no partidarios en respuesta al desprecio habitual de la izquierda hacia sus inquietudes por las relaciones de poder estructuradas en torno al género y la sexualidad, desestimadas por la izquierda en tanto divisorias, burguesas o, en el mejor de los casos, secundarias a la "lucha central", es decir, la lucha de clases (De la Dehesa 2010). Esas raíces explican el peso en las tempranas políticas sexuales en la región de los debates superpuestos sobre intersecciones entre feminismo y liberación sexual con la lucha de clases, y sobre las relaciones que los movimientos sociales emergentes deberían establecer con la izquierda, los partidos políticos y el Estado.
Con las transiciones democráticas, en materia de género y sexualidad, las mujeres fueron las primeras en (re)ingresar a la escena pública, participando en movimientos feministas y movimientos de mujeres más amplios y heterogéneos. En la primera línea de la oposición a los regímenes autoritarios, las mujeres también se movilizaron a través de los organismos de derechos humanos, de familiares de desaparecidos y víctimas de la represión, así como de ollas populares, comunidades de base y organizaciones barriales con trabajo en servicios básicos de salud, guardería o vivienda. Adelantándose al desarrollo de una noción de "derechos sexuales", las feministas impulsaron entonces legislaciones contra la violencia sexual y doméstica, cambios en los códigos civiles para permitir el divorcio $\mathrm{y}$ establecer relaciones de género más igualitarias en el seno del matrimonio, y el derecho a una "maternidad libre $\mathrm{y}$ voluntaria". Reflejando sus articulaciones con la izquierda, las primeras feministas latinoamericanas estuvieron atentas a la relación entre la lucha contra el patriarcado y la lucha anti-imperialista. Tanto a nivel nacional como internacional, las feministas latinoamericanas cuestionaban las políticas demográficas neomalthusianas, apoyadas por las agencias internacionales de desarrollo, que reducían a las mujeres a su capacidad reproductiva y justificaban por 
ejemplo políticas de esterilizaciones involuntarias masivas. Desde el feminismo se promovió en cambio, la atención integral de la salud de las mujeres (Sternbach, Navarro-Aranguren, Chuchryk, \& Álvarez, 1992).

Aun encontrando fuertes resistencias por parte de las feministas heterosexuales (y algunas lesbianas), las feministas lesbianas en particular alentaron discusiones sobre la importancia de combatir el estigma sexual y de valorizar el placer sexual más allá de la reproducción. Por ejemplo, la organización de un caucus de lesbianas y varios talleres sobre lesbianismo en la conferencia paralela a la Primera Conferencia sobre la Mujer de Naciones Unidas, llevada a cabo en México en 1975 (evento que dio ímpetu a los movimientos feministas en varios países), constituyeron el único ámbito en dicho foro en que se discutió algo sobre sexualidad (Bunche \& Hinojosa, 2000).

Al cabo de los años, a medida que los movimientos feministas fueron articulando lazos con los nuevos estados democráticos, comenzaron a pelear por el establecimiento de institutos y programas para las mujeres (aun cuando en muchos casos éstos resultaron poco efectivos y sin recursos), así como por cuotas electorales en las listas partidarias, cuotas que existen en al menos once países de la región (Htun, 2004). Como observara Gloria Careaga en el Diálogo mencionado (Correa \& Parker, 2011), las articulaciones de feministas con otros sectores y causas políticas fueron cediendo en el transcurso de los años, en tanto el paradigma transnacional de la salud sexual y reproductiva ha estrechado las demandas hacia políticas sectoriales específicas por acceso a la planificación familiar y el aborto. Esto ha tenido efectos despolitizadores.

Paralelamente, movimientos gays y lésbicos emergieron en la región, comenzando con el establecimiento del Grupo Nuestro Mundo en Argentina en 1969 (Brown, 1999). A menudo despreciados, al igual que el feminismo, por "foráneos" o pequeño-burgueses, tanto por la derecha como por la izquierda, los primeros militantes abrevaron en un ethos contracultural para promover la revolución de las normas sexuales. Como lo expresaban los participantes en 1979 de la Marcha del Orgullo Gay Lésbico en la Ciudad de México, la primera de la región: "No hay liberación política sin liberación sexual". Con la consolidación de las democracias formales, estos movimientos también fueron abandonando las primigenias aspiraciones revolucionarias, rebautizados como movimientos LGBT con discursos de derechos humanos y civiles. Así, la Comunidad Homosexual Argentina, en 1983, proclamaba: "La 
sexualidad es un derecho humano" (Petracci \& Pecheny, 2007).

Progresivamente, militantes travestis, transexuales y/o transgénero, presentes en algunos movimientos desde el principio, formaron las primeras organizaciones trans en la región hacia los años 1990. Avanzando más que otros movimientos sociales en cuestionar el binarismo de género dominante, sus organizaciones priorizaron la necesidad de terminar con la violencia transfóbica y el abuso policial, acceder a la prevención y tratamientos del VIH/sida, sancionar leyes de identidad de género que permitan la adecuación del nombre de pila y el sexo en los documentos oficiales, garantizar el acceso a los tratamientos de adecuación corporal y genital (ambas obtenidas en Argentina en 2012), así como el acceso al empleo, la educación y la salud.

La epidemia del VIH/sida, con impacto en gays/hombres que tienen sexo con hombres y travestis de toda la región, ha tenido profundos efectos en la militancia LGBT y el modo de configurar los reclamos por derechos. Aunque al inicio de la epidemia se temiera y se observara el refuerzo de estigmas sociales de larga data en relación con la sexualidad, la epidemia aumentó el imperativo del activismo a comprometerse con el Estado, al tiempo que les abrió el paso hacia los ministerios de salud y programas gubernamentales de VIH/sida para el avance de reivindicaciones de derechos. Gays y travestis adquirieron de este modo "terapéutico" unos primeros derechos de ciudadanía.

En el marco de estos procesos de medicalización de la política sexual en la región (Pecheny, 2010), las lesbianas, consideradas como una población menos vulnerable al VIH, permanecieron invisibles para las políticas públicas en la mayoría de los países, y se encuentran todavía hoy marginadas de la política pública y el financiamiento, reforzando de hecho la división histórica generizada entre público y privado.

También se formaron en casi todos los países de América Latina organizaciones de defensa de los derechos en relación con el trabajo sexual y la situación de prostitución (luego también sobre trata y tráfico), atravesando diversas identidades de género así como modos de conceptualizar a las personas y a la propia actividad. En esta última ola de democratización, el primer grupo sociopolítico de trabajadoras sexuales se formó en Ecuador en 1982, la Asociación Autónoma de Mujeres Trabajadora "22 de junio", organización que logró captar la atención nacional dos años más tarde cuando llamaron a una huelga de protesta contra el abuso policial y la explotación por burdeles y proxenetas (Abad, Briones, 
Cordero, Manzo, \& Marchán, 1998). El movimiento creció en tamaño y cobertura geográfica desde los años 1990, nuevamente, en parte como reflejo del impacto del financiamiento $\mathrm{y}$ reconocimiento del trabajo preventivo en VIH/sida. En 1997, en un encuentro en Costa Rica se constituyó la RedTraSex, una red regional de organizaciones de trabajadoras sexuales que representa al menos quince países. Con un discurso de derechos humanos, las militantes han priorizado la des-estigmatización del trabajo sexual, el reconocimiento de los derechos laborales para la profesión, el combate al abuso y violencia policiales, y la prevención del VIH/sida. En América Latina existe hoy un debate apasionado y argumentado en torno a la oposición entre trabajo sexual y situación de prostitución (en casos, identificada a su vez con la trata/tráfico). Lo que está en juego en las discusiones es en qué medida debe respetarse la elección de entrar a esta actividad y defenderse el mejoramiento de las condiciones de trabajo, o si esto no es una manera de reproducir un sistema injusto y a lo que debe apuntarse es a cambiar los arreglos estructurales que condicionan tal elección y desarrollar alternativas para otras profesiones, lo cual es criticado por reforzar de alguna manera el estigma ligado al trabajo sexual. Las diferencias políticas en la materia han creado en algunos contextos una distancia entre el movimiento de trabajadoras sexuales y gran parte de las feministas de la región. A su vez, los condicionamientos estructurales en el marco de las industrias del sexo afectan más fuertemente a las travestis que a las mujeres, aunque el imperativo de intervenir en las condiciones de acceso diferencial a la educación y al mercado de trabajo afecta al conjunto de personas que realizan trabajo sexual. En otro orden de cosas, las dimensiones geopolíticas y neo-imperialistas del asunto suelen ser olvidadas en las discusiones sobre sexo comercial y migraciones más o menos forzadas o voluntarias, temporarias o permanentes.

Estas cuestiones que cruzan sexualidad y economía se convirtieron en "cuestiones políticas calientes" en la región. Sin embargo, más allá del trabajo sexual que con evidencia integra ambas dimensiones, es preciso investigar $\mathrm{y}$ discutir más para dar cuenta políticamente de las dimensiones sexuadas de las relaciones económicas y las dimensiones económicas de todas las relaciones sexuales, la economía sexual del "sistema político y social" en su conjunto.

Finalmente, como dijimos, desde que los primeros casos de infección de VIH fueran reportados en la región a inicios de los años 1980, el impacto de la epidemia en la militancia y política 
sexuales ha sido profundo. Además de reorientar las actividades de muchas organizaciones LGBT, de trabajadoras sexuales, y también feministas, hacia el trabajo preventivo, se desarrolló un extenso movimiento específico en torno al VIH/sida incluyendo acompañamiento, prevención y defensa de derechos, investigación y educación. Las organizaciones y redes de personas viviendo con VIH han jugado un papel central en lograr el acceso universal a las terapias antirretrovirales y obligando a los Estados, no sin conflictos, a ocuparse de los tratamientos y la salud integral de las poblaciones afectadas. Siguiendo modelos de gestión promovidos por las agencias internacionales, varios gobiernos incorporaron por primera vez organizaciones de la sociedad civil a las instancias de políticas públicas a través de sus programas nacionales y locales, de modo tal que en todos lados se dieron colaboraciones multisectoriales. Para las categorías más estigmatizadas, como las trabajadoras sexuales y las travestis, que los partidos políticos y legisladores consideran a priori irrepresentables, el sector salud se convirtió en muchos casos en la principal o única puerta de entrada para comprometer al Estado con sus situaciones. Los nuevos financiamientos y circulación trasnacional incrementaron la fuerza y visibilidad de los movimientos sociales sobre VIH/sida, aunque ello tuvo sus costos. Esta integración alentó aquellos modos de militancia orientados por lo que Jane Galvão (2000) llamó “dictadura de los proyectos", modos que limitan los horizontes temporales a la duración de los financiamientos, contribuyen a la competencia centrífuga entre activistas y refuerzan la medicalización de la política sexual, en modos similares a lo que produjo el giro internacional del feminismo hacia el privilegio del lenguaje de la "salud sexual y reproductiva" como base de la legitimidad de las reivindicaciones.

\section{Escenarios cambiantes de la militancia social y la representación política}

Sin negar la especificidad de cada movimiento y las múltiples diferencias según los países y localidades, es posible identificar tendencias que han caracterizado el curso de la política sexual en la región en su conjunto a lo largo del tiempo. Organizativamente, todos han experimentado, en mayor o menor medida, procesos paralelos de profesionalización, ONGización y creciente trasnacionalización.

Las ONG pueden jugar un papel importante en incidencia política (advocacy), investigación y provisión de servicios. En tanto brokers de información, traducen el conocimiento experto médico, 
legal $\mathrm{y}$ otros en lenguaje ordinario $\mathrm{y}$ accesible para las poblaciones con las que trabajan, al tiempo que proveen a los otros actores involucrados ("stakeholders") de información sobre las cuestiones $\mathrm{y}$ poblaciones que representan. Aun cuando juegan un papel cada vez más importante en la gobernancia de las democracias latinoamericanas, en parte compensando las brechas y limitaciones de la acción estatal en el contexto contemporáneo de globalización, las ONG no son en sí mismas democráticas en su organización, y de hecho operan dentro de redes que valorizan (presuntamente de modo apolítico, en todo caso, por definición no partidario) las capacidades técnicas. $\mathrm{Su}$ creciente importancia en la sociedad civil plantea así nuevas cuestiones sobre los modos y naturaleza de la representación política, así como de la rendición de cuentas (accountability) y fidelidades sociales, cuestiones que, junto con la competencia por el acceso a los fondos, han promovido a veces las divisiones dentro de los movimiento sociales (Clark, 2003; Ramos, 2004; Silva, 1998; Álvarez, Danigno \& Escobar, 1997).

El proceso de ONGización es coherente con los cambios en la arena internacional, que incluyen el peso creciente de las redes trasnacionales de activismo y gobernancia. Es cierto que los movimientos sociales han mantenido históricamente articulaciones muy variadas, pero la envergadura y frecuencia de tales contactos se incrementaron en las décadas y los años recientes. Una expresión particularmente importante de este fenómeno han sido las conferencias y Encuentros feministas latinoamericanos y del Caribe, que tienen lugar regularmente desde 1981. Estos espacios fueron una instancia importantísima para la creación y desarrollo de lo que Nancy Fraser (1994) denominó "contra-públicos subalternos", espacios deliberativos en los que actores subordinados pueden acumular recursos simbólicos, articular políticamente, y desarrollar nociones alternativas de identidad y justicia. Transformaciones profundas en el movimiento feminista se fueron dando en estos eventos, arenas de conflicto tanto como de consenso. Esto se refleja, por caso, en la insistencia de las organizadoras de los primeros encuentros en auto-financiarse completamente, mientras que luego se buscaron fuentes externas; debates subsecuentes entre las llamadas institucionalizadas y autonomistas se focalizaron de algún modo en las implicancias de la ONGización; o la inclusión del lesbianismo o del aborto como temas centrales de la política feminista (Álvarez y otros, 2002; Sternbach, Navarro-Aranguren, Chuchryk, \& Álvarez, 1992). 
Otro espacio que alentó articulaciones trasnacionales sobre sexualidad, esta vez ligado desde el primer momento a la salud y los modos de gobernanza globalizados, fue el Grupo de Cooperación Técnica Horizontal (GCTH). Establecido en 1995, reunía inicialmente a los directivos de 21 Programas Nacionales de VIH/sida de la región, pensado como contrapeso de las agencias internacionales con trabajo en sida. Desde entonces, el GCTH incorporó y contribuyó a formar diez redes regionales latinoamericanas de poblaciones vulnerables de la sociedad civil, incluyendo, por ejemplo, la RedTraSex ya mencionada, la Asociación para la Salud Integral y Ciudadanía de América Latina y el Caribe (ASICAL fundada en 1997 y que reúne organizaciones que trabajan con gays $\mathrm{y}$ "hombres que tienen sexo con hombres") y la Red Latinoamericana y del Caribe de Personas Trans (RedLacTrans -creada en 2004 y que reúne travestis, transexuales y transgéneros). Además de esos espacios y redes regionales, las Conferencias y postConferencias de Naciones Unidas sobre Derechos Humanos, Población y Desarrollo, y de la Mujer han sido instancias de movilización no gubernamental en toda la región.

Tanto la importancia creciente de las redes trasnacionales como la ONGización del activismo hablan de una reconfiguración más profunda tanto de la gubernamentalidad como de la representación política en América Latina. El descentramiento (aunque no el desplazamiento) del Estado-nación como principal sitio del poder de decisión ha ido de la mano del desarrollo de redes que articulan lazos no sólo entre ONG sino también con actores estatales al nivel local y nacional, con instituciones semi-estatales o para-estatales, agencias internacionales, fundaciones, academia, empresas privadas, etc., incorporándose tanto a la formulación como a la implementación de políticas. En relación con la dispersión del poder de toma de decisiones supuestamente reflejada en estos cambios, las redes rompen con modos más horizontales $\mathrm{y}$ democráticos-populistas de representación política que históricamente han prevalecido en la región. Mientras que los líderes populistas y de la izquierda privilegiaron las organizaciones de masas estructuradas primariamente en torno de la clase social y las identidades populares, resulta más probable que las redes emergentes se movilicen en torno de grupos identitarios o áreas de políticas específicas, y se caracterizan por la fluidez de su composición y habilidad para reunirse en torno de campañas coyunturales. En verdad, la internacionalización de las políticas públicas y el descentramiento de los 
procesos de toma de decisiones implicados por esta reconfiguración han creado, para los militantes, oportunidades de ejercer presión política a través de canales alternativos, cuando el acceso al Estado está cerrado para ellos. Pero en el contexto de democracias emergentes atravesadas por fórmulas neoliberales de gubernamentalidad, las lógicas tecnocráticas de las políticas públicas coincidieron en este proceso que terminó por socavar los mecanismos de la rendición de cuentas democrática (Montecinos, 2001). Como señalara la historiadora Elsa Muñiz (2011: 109), cuando el Estado era considerado el principal responsable de la salud de la población, al menos tenía una cara identificable; con el advenimiento de los "estados esquizofrénicos neoliberales", dicha responsabilidad se diluye en una multiplicidad de actores, y el camino hacia la articulación del conflicto y los reclamos, necesario para la apropiación efectiva de derechos, no está siempre claro. Además, aunque tales redes pretendan en un sentido ideales de horizontalidad y deliberación, no están para nada exentas de asimetrías de poder (Chalmers, Martin, \& Piester, 1997). Tales dinámicas condicionan no sólo las relaciones entre actores en las redes (entre grupos y ONG con capacidades variables, y entre las ONG y las otras instituciones con las que articulan, en particular las financiadoras), sino también los mecanismos de selección que permiten el propio acceso a las redes, desde los requerimientos formales hasta los imperativos informales como la capacidad de financiar viajes. Dicho esto, es en las esferas públicas nacionales en las que el Estado-nación mantiene su lugar central en el imaginario político, por lo cual puede preverse un regreso del Estado y, quizá, a la vez un avance en la inclusión política acompañado de un disciplinamiento de las organizaciones de la sociedad civil.

Los cambios en la organización de la sociedad civil y en las arenas políticas más amplias que transitan los militantes han contribuido a transformaciones en sus estrategias políticas y discursivas. Las críticas marxistas al patriarcado y la familia, las celebraciones liberacionistas del placer y la transgresión sexual, los reclamos políticos por el cuerpo, alguna vez tan centrales para la política sexual en la región, se encuentran ahora algo marginados, en la medida en que la lingua franca de los derechos humanos y la salud sexual y reproductiva prevaleció en las esferas públicas nacionales e internacionales. Esos cambios permitieron a los militantes establecer puentes con los actores estatales, las agencias internacionales y los partidos políticos, en especial de la izquierda (que a su vez se fue transformando en estos años); pero ello 
contribuyó también a la medicalización del lenguaje activista y a la contención reformista de la imaginación política, de modo que muchas vías alternativas y con pretensiones revolucionarias se volvieron impensables.

Teniendo en cuenta estos procesos cabe interrogarse, también, acerca de las limitaciones y los costos de las políticas de identidad que se han desarrollado en la región en los últimos años. Desde las teorías de la interseccionalidad (Crenshaw, 1991; Collins, 2002; Marcos, 2003; Moraga \& Anzaldúa, 1983; Viveros Vigoya, 2002), se alerta sobre el peligro de que la reificación de las identidades como fundamento naturalizado de la acción política pueda oscurecer y violentar las dinámicas internas e imposiciones de poder que existen al interior de categorías como las de mujer, gay, trabajadora sexual, etc. Una mirada interseccional (Gil Hernández, en Correa \& Parker, 2011) permitiría prestar atención tanto a las relaciones de dominación masculina y/o heterosexista dentro de comunidades marginalizadas o subordinadas, como a las dinámicas coloniales que pueden vehiculizarse a través de discursos que se reclaman universales, especialmente el de los derechos humanos o el de la salud (Ayres, 2002).

Este tipo de discusiones han sido relevantes para los movimientos sociales de la región desde los tempranos debates sobre intersecciones entre opresiones de género, sexuales y de clase. En las conferencias $\quad y \quad$ encuentros latinoamericanos feministas y de mujeres, las militantes lesbianas, indígenas y negras cuestionaron el racismo y el heterosexismo dentro del movimiento feminista, subrayando la necesidad de articular las luchas contra las múltiples formas de opresión, y las llevaron por ejemplo a la organización de encuentros regionales de lesbianas, convocados regularmente desde 1987. El Primer Encuentro de Mujeres Negras Latinoamericanas y del Caribe tuvo lugar en la República Dominicana en 1992, como una alternativa a la celebración de la conquista europea de 1492, en el cual más de 300 representantes de 32 países crearon su propia red regional. Asimismo, en respuesta a la falta de representación en el encuentro preparatorio para la Conferencia de Beijing, activistas indígenas crearon un mecanismo alternativo de deliberaciones y luego tuvieron un Primer Encuentro Continental de Mujeres Indígenas en 1996 en Quito, donde establecieron una red regional (Alvarez et al., 2002). Más recientemente, en el Segundo Encuentro de Líderes Indígenas y Feministas de Lima en 2008, junto con la Campaña por una Convención Inter-Americana de Derechos Sexuales y Reproductivos, las participantes discutieron las tensiones entre los 
movimientos feministas e indígenas en la región, como por ejemplo el peso relativo dado a los derechos individuales $\mathrm{y}$ colectivos en las respectivas agendas.

Aun reconociendo su importancia, debe subrayarse la dificultad de trasladar estas críticas interseccionales al diseño e implementación de las políticas públicas: numerosos gobiernos nacionales y locales de la región, como el de Bogotá (Gil Hernández, 2011), quisieron dar cuenta de la diversidad y adoptaron una lógica sectorial de gubernamentalidad que reproduce y refuerza el discurso de las políticas de identidad a través de la creación de programas especializados que apuntan a poblaciones determinadas (jóvenes, LGBT, mujeres, comunidades indígenas, etc.). A menudos estos programas se basan en bellas palabras que resultan en escasas acciones, en una lógica que festeja livianamente la nueva celebración trasnacional de la diversidad y las categorías identitarias pero oscurecen las relaciones de poder que las hacen emerger. Mientras que en principio las perspectivas interseccionales podrían abrir posibilidades para políticas de coalición, el resultado suele ser una mayor atomización de los movimientos sociales y una exacerbación de la competencia por la atención pública y política, y el financiamiento.

\section{Sobre los límites del compromiso con el Estado}

Más allá de las transformaciones en la militancia y los movimientos sociales, vale la pena hacerse la pregunta por las limitaciones reales de qué es lo que puede lograrse mediante el compromiso con el Estado. Como dijimos, en el contexto de las transiciones a la democracia en la región, la militancia por los derechos sexuales ha sido capaz de abrirse caminos hacia las esferas públicas y políticas, y lograr victorias formales, con diferencias entre países, ciudades y áreas de políticas. En parte, estos logros expresan la inserción de los derechos sexuales en los discursos más generales de los derechos humanos, el cambio democrático y en los problemáticos discursos teleológicos de la modernización cultural, económica y política. Por otra parte, como llamara la atención Rosalind Petchesky (1999), existe un fuerte consenso internacional en construir a los derechos sexuales más en términos de libertades negativas, de protección ante violencias, abusos y persecuciones, que como derechos positivos, que incluyen derechos al placer y la realización personal. En América Latina, esta tendencia se refleja en el claramente mayor apoyo del espectro político hacia las leyes contra la discriminación, la violencia sexual y doméstica, y la trata o el tráfico, 
que hacia leyes y políticas que reconocen los derechos laborales de las trabajadoras sexuales, la identidad de género, el acceso al aborto legal, y los derechos de las parejas del mismo sexo. Respecto de esto último, sin embargo, pasos significativos hacia el reconocimiento se han dado en los últimos cinco años en Argentina (con la aprobación en 2010 del matrimonio igualitario), Uruguay, Colombia, Brasil, Ecuador y México, a veces gracias al papel clave del sector judicial, lo que sugiere el inicio de un nuevo consenso, nuevamente haciendo eco de los marcos liberales de la igualdad y la universalidad, e incluso de la impronta del lenguaje y la utopía del amor romántico como sustento del reconocimiento de derechos. El reconocimiento del matrimonio igualitario es un salto político cualitativo para la región, como lo implicaría la legalización de la interrupción voluntaria del embarazo.

En este sentido, los nuevos contextos políticos también incluyen una todavía muy influyente iglesia católica, cuyo peso político se vuelve evidente en la cuestión del aborto (Carino, González Vélez \& Guzmán, 2012). Se estima que cuatro millones de mujeres abortan clandestinamente cada año en la región, y el aborto en condiciones inseguras representa una de las principales causa de las muertes maternas y de complicaciones para la salud de las mujeres en edad reproductiva. Sin embargo las legislaciones en América Latina son de las más restrictivas del mundo, con las excepciones de Puerto Rico y Cuba, y algunos pasos hacia la liberalización en Colombia y la Ciudad de México. La discusión sobre el tema sigue paralizada en la mayoría de los países, como Argentina o Uruguay, e incluso en algunos casos se reforzó la criminalización como en Nicaragua, El Salvador, la República Dominicana y varios estados de México. En las dinámicas electorales, las élites de los partidos buscan el apoyo de la iglesia y otros actores religiosos, o al menos no despertar su enemistad. Si la victoria de la izquierda en varios países de la región muestra un repudio significativo a la hegemonía neoliberal de los años 1990, en cuestiones de derechos sexuales el conservadurismo puede atravesar el abanico izquierdaderecha, particularmente con referencia al aborto. Estas dinámicas, por ejemple, fueron evidentes en el endurecimiento de la legislación sobre aborto en Nicaragua. Justo antes de la elección de 2006, el congreso, dominado por el supuestamente izquierdista Frente Sandinista de Liberación Nacional y el Partido Constitucionalista Liberal (de derecha), votaron por unanimidad abolir en el código penal de 1893 el permiso a abortar cuando el embarazo representa un riesgo para la salud de la mujer. A pesar de las demandas 
feministas, Ministerio de Salud y comunidad médica por posponer la reforma hasta después de la elección, ambos partidos precipitaron el proceso restrictivo con el fin de seducir el favor de la iglesia católica (Montenegro, 2006).

La religión es un aspecto ineludible del contexto actual de democratización y globalización. En América Latina, la iglesia católica es la más influyente políticamente (según el tipo de competencia electoral) y la más conservadora (según las políticas cambiantes del Vaticano); a ella se suma un panorama religioso más heterogéneo (evangélicos y protestantes representan en diez países más del 15 por ciento de la población). En algunos lugares, la maquinaria electoral evangélica refuerza la oposición a los derechos sexuales (como la bancada evangélica en Brasil); en otras instancias, pueden apoyar medidas de derechos sexuales (por ejemplo, algunos líderes y denominaciones protestantes apoyaron el matrimonio igualitario en la Argentina). El activismo religioso es cada vez más trasnacional, articulando lazos al nivel local. Dichas tendencias encontraron respuestas desde la militancia sexual, principalmente la defensa del secularismo y la laicidad. Esta estrategia, más efectiva en algunos países que en otros (México, por ejemplo, tiene una larga historia de separación de la Iglesia y el Estado), plantea algunos dilemas políticos: el riesgo de alejar a potenciales públicos y aliados religiosos y de etiquetar a las comunidades religiosas como retrógradas (reiterando así las narrativas progresistas de la modernización). Por su parte, los sectores religiosos se están adaptando a los nuevos contextos, defendiendo un "secularismo estratégico" y planteando así nuevas cuestiones y desafíos a los movimientos sociales (Vaggione, 2011, en Correa \& Parker 2011).

Por último, incluso cuando los derechos sexuales están formalmente instituidos, la militancia de la región enfrenta el viejo dilema derivado de las brechas y distancias entre la letra de las leyes, la implementación de políticas, y las prácticas sociales. Este fenómeno asume varias formas, desde la existencia de legislación represiva que es implementada selectivamente, hasta derechos reconocidos formalmente que se quedan en la letra escrita. En el caso del aborto, por ejemplo, la distancia entre su criminalización y la extendida práctica clandestina refuerza y supone otras desigualdades. Mientras que las mujeres de clases medias y superiores acceden a procedimientos clandestinos pero seguros, las más pobres y marginadas corren riesgos serios para su salud y su vida. Esto trae consecuencias políticas. Las mujeres con más recursos y por ende con una mayor capacidad de intervenir 
políticamente evitan sin grandes inconvenientes las consecuencias graves de la criminalización (no aplicada, pero potencialmente aplicable) del aborto; eso reproduce los dobles discursos y debilita la potencial "base" para la presión política por la liberalización del aborto. La aplicación selectiva de las regulaciones y restricciones al trabajo sexual, en otro orden, también alienta los dobles estándares socio-económicos, y los arreglos informales, inseparables de la corrupción y abuso de la policía.

Por otro lado, las leyes y políticas que presuntamente favorecen los derechos sexuales a menudo no son implementadas o no son usadas por los sujetos, dadas las barreras y condiciones materiales $\mathrm{y}$ simbólicas para el ejercicio de los derechos formales y de los programas existentes. Este es el caso, muchas veces, de la legislación anti-discriminatoria, que a menudo equivale más a la simple afirmación de ideales que a un instrumento legal que puede ser usado; o de las normativas que reconocen a las parejas del mismo sexo, cuando el estigma de la homosexualidad obliga a muchos individuos a permanecer en la discreción; o la protección en el empleo ante el acoso y la arbitrariedad en relación con la sexualidad, o en los casos de violación. Las razones de este fenómeno son de diverso orden. Algunas leyes están redactadas de modo tal que hacen difícil o imposible su apropiación por parte de los sujetos, o las condiciones de acceso para algunos programas y políticas plantean obstáculos imposibles de sortear. La adecuación documentaria de las personas trans, por ejemplo, a menudo exige un diagnóstico patológico de trastorno de identidad de género, o incluso la realización previa de una cirugía de modificación genital - la cual puede implicar, entre otras cosas, la esterilidad. Se pide entonces un certificado de enfermedad o una mutilación para que el Estado reconozca el acceso a derechos, y a su vez presupone que estos sujetos - aun aceptando brutal e instrumentalmente esos requisitos - tienen asesoramiento legal y acceso al sistema de salud. En numerosos casos son los conflictos entre jurisdicciones los que impiden el acceso a bienes y servicios garantizados por las leyes y políticas, como en el caso de la anticoncepción de emergencia en Chile, cuyo acceso la presidenta Michelle Bachelet garantizó por decreto en 2006 para las mayores de 14 años, y que autoridades locales ignoraron; o históricamente, en todos lados, la provisión de preservativos y anticonceptivos a adolescentes, jóvenes, etc.

Las brechas entre leyes, políticas y prácticas son indicativas de una relación más general entre el Estado y la sociedad, reflejada en formas estratificadas de 
ciudadanía y una relativamente superficial penetración de la esfera pública en muchos países y partes de países de la región (Avritzer, 2002). Como resultado tanto de las débiles capacidades estatales como de la falta de voluntad política, en vastas áreas rurales y urbanas de marginación, la presencia estatal se limita a su aparato represivo y de seguridad, o directamente el Estado está ausente; se trata de espacios donde los derechos de ciudadanía más básicos están suspendidos o muy distorsionados (O’Donnell, 1993). En estos espacios, hay endémicas disputas entre las débiles instituciones estatales $\mathrm{y}$ gubernamentales, las empresas privadas, los grupos vinculados a caciques y caudillos locales, ONG, iglesias, narcos, guerrillas y fuerzas paramilitares. Los diversos grados y calidades de la presencia estatal democrática instituye así condiciones diferenciales, y aun opuestas, en términos, por ejemplo, de los derechos LGBT, como en Colombia y el contraste existente entre las acciones y logros de la ONG Colombia Diversa, materializados en los principales centros urbanos, y la violencia homofóbica y sexista que se ha dado en las zonas controladas por las guerrillas y grupos paramilitares en nombre de la "limpieza social" (Gallegos, en Correa \& Parker, 2011).

En suma, para la militancia sexual, estas realidades plantean al menos un doble desafío. Por un lado, muestran los límites a las perspectivas de política sexual centradas en el Estado y de las victorias formales que el activismo generalmente cita cuando proclama sus logros ante los financiadores, agencias estatales y público en general, sugiriendo que un curso diferente pudiera ser necesario para cambiar las actitudes y prácticas en el nivel de la vida cotidiana. Por otro lado, es necesario repensar los procesos de apropiación subjetiva de los derechos (Amuchástegui \& Rivas, 2008), es decir del uso de los derechos y las políticas por parte de los diversos sujetos.

\section{Conclusiones}

Para recapitular, América Latina es un continente lleno de paradojas y tensiones. Políticamente, los movimientos de derechos sexuales en la región reemergen en un paradójico momento histórico de democratización política y reformas liberales. Esto ha producido una reorientación de la oposición frontal ante el Estado, a trabajar con y dentro del mismo. En el período que va desde los años ochenta hasta el presente, nuevas fuentes de financiamiento y dinámicas que van más allá de la región latinoamericana, contribuyeron a la profesionalización y ONGización de los movimientos sociales, 
una transformación coherente con las lógicas neoliberales.

Las respuestas del Estado y los partidos varían de acuerdo con las realidades locales y los temas y sujetos, pero puede reconocerse una tendencia de progreso a lo largo de la región. Algunos partidos, generalmente de izquierda pero también populistas, asumieron la causa de los derechos sexuales. El momento histórico ha permitido avances, en parte por la resonancia de los derechos sexuales con discursos más amplios de derechos humanos, democracia, modernidad liberal y reformismo populista, así como del lenguaje medicalizado de la salud (sexual y reproductiva, y $\mathrm{VIH} /$ sida).

La dinámica electoral se revela como un arma de doble filo, en la medida en que la iglesia católica y otras religiones organizadas son en algunos países un importante contrapeso con poder (real o percibido como tal) de veto en elecciones polarizadas, lo cual termina reproduciendo un estatus quo de restricciones de derechos y/o de dobles estándares y discursos. En relación con el reconocimiento de derechos sexuales, las arenas no partidarias o estatales no electorales, como las cortes o burocracias del sistema de salud, probaron ser una alternativa a los poderes legislativos y ejecutivos.

Hay muchas limitaciones $y$ cuestiones pendientes, y los avances son desparejos según los países y áreas de políticas. El aborto es la cuestión pendiente más importante, mientras que el reconocimiento de parejas del mismo sexo parece ser uno de los "grandes éxitos" en algunos países, como Argentina. Por otro lado, los derechos reconocidos formalmente a menudo se quedan en el papel, debido a la falta de voluntad política y a que hay extensas zonas de excepción, ausencia estatal y/o "zonas marrones" de democracia (O’Donnell, 1993). La construcción de la sexualidad, como cuestión política y de políticas públicas, refleja además la tensión entre una configuración (framing) que es política y sexual, y una que es apolítica (o incluso anti-política) y asexual. Los discursos científicos y expertos juegan un papel clave en este proceso de despolitización y des-sexualización, como lo señalan las críticas epistemológicas y políticas, fundamentadas en epistemologías feministas y del Sur, que plantean diferentes puntos relativos a la naturaleza "situada" del conocimiento, a las nociones contextuales de evidencia, y a la confluencia de discursos dominantes de la biomedicina y de los patrones normativos y científicos del Norte.

Para concluir, llamamos la atención sobre tensiones y perplejidades, en forma interrogativa. Cuestiones relativas a la construcción de coaliciones y demandas de 
políticas que contemplen intersecciones de raza, etnicidad, género, sexualidad y clase social, alientan a los actores a pensar y actuar políticamente. La política (conflictiva) y las políticas (institucionalizadas) tienen a menudo lógicas diferentes: resistir la lógica atomizante y competitiva del lenguaje e instituciones de políticas públicas es un acuciante desafío para la diversidad de actores en la arena sexual; sobre todo en un contexto en el cual son frecuentes las competencias de juego suma-cero entre cuestiones, entre actores, y entre agendas urgentes y estructurales. Las acciones pero también los análisis - son complejos en estas sociedades estado-céntricas, si consideramos que a menudo los Estados "no funcionan" o no están presentes. Esto plantea cuestiones de gubernamentalidad, regulación y hegemonía - para usar conceptos fecundos que remiten a autores y paradigmas diversos, y no fácilmente articulables. Brechas, discontinuidades y disparidades parecen ser el patrón común a lo largo de la región latinoamericana, junto con el reconocimiento de la naturaleza no neutral y (aún) no-secular del Estado: un Estado que sigue siendo capitalista y no del todo separado de la Iglesia. Las brechas son tan grandes en la región que el discurso de los derechos sexuales y los derechos humanos podría ser considerado como una "ideología", como un discurso falso que esconde una realidad persistente de injustas y extendidas desigualdades. No obstante, los fenómenos, análisis, tensiones $\mathrm{y}$ perplejidades ilustradas en este texto, muestran que en América Latina la sexualidad se ha constituido en una arena política vibrante y dinámica.

\section{Notas}

${ }^{1}$ Como señala Brown (2006: 72), la crítica de Foucault de los modelos convencionales de poder y su propia formulación del poder como productivo y disperso, en lugar de represivo y concentrado abre el camino para reconsiderar los modos en que individuos y poblaciones son ordenados $\mathrm{y}$ movilizados en una sociedad de masas, modernamente a la vez unidos en un todo y tratados de manera singular; el gobierno político moderno incluye una combinación no sistemática de micro- y macro-poderes que operan en los cuerpos y mentes de maneras locales y no siempre evidentes, y poderes que pueden ser más abiertos, centralizados y visibles. En América Latina, es nuestra hipótesis, aun las manifestaciones de muchos fenómenos de micro-poderes tienen como escenario, referencia, supuesto, antagonista, y muchos otros papeles, al Estado - descentrado quizá, pero omnipresente. El Estado constituye actores, brinda sentidos, dirime disputas incluso más allá de su propio ámbito formal e informal de intervención. 


\section{Referencias}

Abad, A., Briones, M., Cordero, T., Manzo, R., \& Marchán, M. (1998).

The Association of Autonomous Women Workers, Ecuador: "22nd June". En K. Kempadoo, \& J. Doezema, Global Sex Workers: Rights, Resistance and Redefinition (págs. 172-177). New York: Routledge.

Alvarez, S. E., Dagnino, E., \& Escobar, A. (1997). Cultures of politics, politics of cultures: revisioning Latin American social movements. Boulder: Westview Press.

Alvarez, S. E., Friedman, E. J., Beckman, E., Blackwell, M., Slotz Chinchilla, N., Lebon, N., y otros. (2002). Encountering Latin American and Caribbean Feminisms. Signs: Journal of Women in Culture and Society, 28(2), 537-579.

Amuchástegui, A., \& Rivas, M. (2008). Construcción subjetiva de ciudadanía sexual en México: Género, heteronormatividad y ética. En I. Szasz, \& Salas.

Avritzer, L. (2002). Democracy and the public sphere in Latin America. Princeton, NJ: Princeton University Press.

Ayres, J. R. (2002). Conceptos y prácticas en salud pública: algunas reflexiones. Revista Facultad
Nacional de Salud Pública

(Colombia), 20 (2): 67-82.

Brown, S. (1999). Democracy and difference: the lesbian and gay movement in Argentina. En A. D. Barry, J. W. Duyvendak, A. Krouwel, A. D. Barry, J. W. Duyvendak, \& A. Krouwel (Edits.), The global emergence of gay and lesbian politics: national imprints of a worldwide movement (págs. 110-132). Philadelphia: Temple University Press.

Brown, W. (2006). En J.S.Dryzek, B.Honig \& A.Phillips, The Oxford Handbook of Political Theory (págs. 65-83), Oxford University Press.

Bunche, C., \& Hinojosa, C. (2000). Lesbians travel the roads of feminism globally. New Brunswick, NJ: Rutgers University Center for Women's Global Leadership.

Cano, G. (1987). Congresos feministas en la historia de México. Fem, 11(58), 24-27.

Careaga Pérez, G. (2004). Orientaciones sexuales: alternativas e identidad. En G. Careaga Pérez, \& S. Cruz, Sexualidades diversas: aproximaciones para su análisis (págs. 171-187). México D.F.: H. Cámara de Diputados, LIX Legislatura, UNAM, PUEG y 
Miguel Ángel Porrúa.

Carino, G., González Vélez, A.C. \& Durán, J. (2012). Aborto legal. Regulaciones sanitarias comparadas, Nueva York: IPPF/RHO.

Carrier, J. (1995). De los otros: intimacy and homosexuality among Mexican men. Nueva York: Columbia University Press.

Carrillo, H. (1999). Cultural change, hybridity and male homosexuality in Mexico. Culture, Health and Sexuality, 1(3), 223-238.

Carrillo, H. (2002). The night is young: Sexuality in Mexico in the time of AIDS. Chicago: Chicago University Press.

Chalmers, D. A., Martin, S. B., \& Piester, K. (1997). Associative Networks: New Structures of Representation for the Popular Sectors? En D. A. Chalmers, C. M. Vilas, K. Hite, S. B. Martin, K. Piester, \& M. Segarra (Edits.), The New Politics of Inequality in Latin America: Rethinking Participation and Representation (págs. 543-592). Oxford: Oxford University Press.

Clark, J. D. (2003). Introduction: Civil Society and Transnational Action. En J. D. Clark (Ed.), Globalizing Civic Engagement: Civil Society and Transnational Action (págs. 1-
18). Londres: Earthscan

Publications Ltd.

Clérico, L., \& Aldao, M. (Edits.). (2010). Matrimonio Igualitario en Argentina. Buenos Aires: EUDEBA.

Collins, P. (2002). The sexual politics of black womanhood. En C. L. Williams, \& A. Stein (Edits.), Sexuality and Gender (págs. 193206). Malden, MA: Blackwell Publishing.

Córdova Plaza, R. (1993). De cornudos, dejadas y otras especies: Un estudio de caso sobre sexualidad en el campo veracruzano. En P. Ponce Jiménez, \& M. Báez Landa (Edits.), Tradición y Modernidad en las Identidades (págs. 54-67). Xalapa, Veracruz: Centro de Investigaciones y Estudios Superiores en Antropología Social.

Corrêa, S., \& Parker, R. (Edits.). (2011). Sexualidade e política na América Latina: histórias, interseções e paradoxos. Río de Janeiro: ABIA.

Creenshaw, K. (1991). Mapping the Margins: Intersectionality, Identity Politics, and Violence against Women of Color. Stanford Law Review, 43(6), 1241-1299.

Decena, C. (2008). Tacit Subjects. GLQ: A Journal of Lesbian and Gay Studies, 14(2/3), 339-359. 
Deere, C. D. \& León, M. (2001). Institutional reform of agriculture under neoliberalism: The impact of women's and indigenous movements. Latin American Research Review. 36(2): 31-64.

De la Dehesa, R. (2010). Queering the Public Sphere in Mexico and Brazil: Sexual Rights Movements in Emerging Democracies. Durham, NC: Duke University Press.

Dore, E. (2000). One step forward, two steps back: Gender and the state in the long nineteenth century. En E. Dore, \& M. Molineux (Edits.), Hidden Histories of Gender and the State in Latin America (págs. 3-32). Durham, NC: Duke University Press.

Foucault, M. (2004). Sécurité, Territoires, Population. París: Gallimard y Seuil.

Fraser, N. (1994). Rethinking the public sphere: A contribution to the critique of actually existing democracy. En H. Giroux, \& P. McLaren (Edits.), Between Borders: Pedagogy and the politics of cultural studies (págs. 74-98). Londres: Routledge.

Galvao, J. (2000). AIDS no Brasil: A agenda de construção de uma epidemia. San Pablo: Editora 34.

Gil Hernández, F. (2011). Estado y procesos políticos: sexualidad e interseccionalidad. En S. Corrêa, \& R. Parker (Edits.), Sexualidade e política na América Latina: histórias, interseções e paradoxos (págs. 80-99). Río de Janeiro: ABIA.

González-López, G. (2005). Erotic journeys: Mexican immigrants and their sex lives. Berkeley: University of California Press.

Heilborn, M. L. (1996). Ser ou estar homossexual: dilemas de construção de identidade social. En M. B. Regina, \& R. Parker (Edits.), Sexualidades pelo avesso: Direitos, identidades e poder (págs. 136145). San Pablo: Editora 34.

Htun, M. (2004). Is gender like ethnicity? The political representation of identity groups. Perspectives on Politics, 2(3), 439-458.

Lascoumes, P. \& Le Galès. (2007). Sociologie de l'action publique, París: Armand Colin.

Lau Jaiven, A. (2009). Entre ambas fronteras: La búsqueda de la igualdad de derechos para las mujeres. Política y Cultura(31), 235-255.

List Reyes, M. (2004). La diversidad sexual vista por la antropología. En G. Careaga, \& S. Cruz (Edits.), Sexualidades diversas: 
Aproximaciones para su análisis (págs. 97-127). México, D.F.: H. Cámara de Diputados, LIX Legislatura, UNAM, PUEG y Miguel Ángel Porrúa.

List Reyes, M. (2005). Jóvenes corazones gay en la Ciudad de México. Puebla: Benemérita Universidad Autónoma de Letras, Facultad de Filosofía y Letras.

Loyola, M. A. (2000). A antropologia da sexualidade no Brasil. Physis: Revista de Saúde Coletiva, 10(1), 143-167.

Marcos, S. (2003). Identidades en transformación: Las prácticas feministas en el movimiento de mujeres indígenas. En P. Bonfil Sánchez, \& E. R. Martínez Medrano (Edits.), Diagnóstico de la discriminación hacia las mujeres indígenas (págs. 1-40). México, D.F.: CDI.

Miano, M., \& Giglia, Á. (2001). Identidades en la construcción y deconstrucción: una exploración del arquipiélago lésbico-gay desde la historia oral. Cuicuilco: Revista de la Escuela Nacional de Antropología e Historia, 8(23), 6795.

Molineux, M. (2000). Twentieth-Century State formations in Latin America. En E. Dore, \& M. Molineux
(Edits.), Hidden Histories of Gender and the State in Latin America (págs. 33-84). Durham, NC: Duke University Press.

Montecinos, V. (2001). Feminists and Technocrats in the Democratization of Latin America: A Prolegomenon. International Journal of Politics, Culture, and Society, 15(1), 175-198.

Montenegro, S. (2006). Nicaragua: el aborto en la batalla electoral del 2006. Debate Feminista, 17 (34), 158-161.

Moraga, C., \& Anzaldua, G. (Edits.). (1983). This bridge called my back: Writings by radical women of color. Nueva York: Kitchen Table, Women of Color Press.

Muñiz, E. (2011). Los puntos conflictivos en la relación entre los Estados y las políticas sexuales. En S. Corrêa, \& R. Parker (Edits.), Sexualidade e política na América Latina: histórias, interseções e paradoxos (págs. 100-110). Río de Janeiro: ABIA.

Nuñez Noriega, G. (1999). Sexo entre varones: Poder y resistencia en el campo sexual. México, D.F.: Coordinación de Humanidades, Programa de Estudios de Género, Universidad Autónoma Metropolitana de México. 
O’Donnell, G. (1993). Estado, democratización y ciudadanía, Nueva Sociedad, 62-87.

Parker, R. (1986). Masculinity, femininity, and homosexuality: On the anthropological interpretation of sexual meanings in Brazil. Journal of Homosexuality, 11(3/4), 155163.

Parker, R. (1995). Hacia una economía política del cuerpo: Construcción de la masculinidad y la homosexualidad masculina en Brasil. En T. Valdés, \& J. Olavarría (Edits.), Masculinidades y Equidad de Género en América Latina (págs. 106-129). Santiago de Chile: FLACSO.

Parker, R. (1999). Beneath the Equator: Cultures of desire, male homosexuality and emerging gay communities in Brazil. Nueva York: Routledge.

Pecheny, M. (2010). Political agents or vulnerable victims? Framing sexual rights as sexual health in Argentina, in R.Parker \& P.Aggleton (eds), Handbook on Sexuality, Health and Rights, Londres: Routledge.

Pecheny, M., \& de la Dehesa, R. (2010). Sexualidades y políticas en América Latina: el matrimonio igualitario en contexto. En L. Clérico, \& M. Aldao (Edits.),
Matrimonio Igualitario en Argentina (págs. 7-58). Buenos Aires: EUDEBA.

Pecheny, M., \& de la Dehesa, R. (2011). Sexualidades y políticas en América Latina. En S. Corrêa, \& R. Parker (Edits.), Sexualidade $e$ política na América Latina: histórias, interseções e paradoxos (págs. 31-79). Río de Janeiro: ABIA.

Petracci, M. \& Pecheny, M. (2007). Argentina: Derechos humanos $y$ sexualidad. Buenos Aires: CEDESCLAM.

Ramos, S. (2004). O papel das ONGs na construção de políticas de saúde: a Aids, a saúde da mulher e a saúde mental. Ciência e saúde coletiva, 9(4), 1067-1078.

Rouquié, A. (1987). Amérique latine. Introduction a l'Extrême-Occident. París: Seuil.]

Silva, C. (1998). ONG/Aids, intervenções sociais e novos laços de solidariedade social. Cadernos de Saúde Pública, 14(2), 129-139.

Stepan, N. L. (1991). "The hour of eugenics": Race, gender and nation in Latin America. Ithaca: Cornell University Press.

Sternbach, N. S., Navarro-Aranguren, M., Chuchryk, P., \& Álvarez, S. E. (1992). Feminisms in Latin 
America: From Bogotá to San

Bernardo. Signs, 17(2), 393-434.

Viveros Vegoya, M. (2002). Dionysian

blacks: Sexuality, body, and racial order in Colombia. Latin American Perspectives, 29(2), 60-77.

Mario Pecheny: Doctor en Ciencia

Política, es Profesor de Ciencia Política y Sociología de la Salud en la Universidad de Buenos Aires e investigador del Consejo Nacional de Investigaciones Científicas y Técnicas (Argentina), pecheny.mario@gmail.com

Rafael de la Dehesa: $\mathrm{Ph} \mathrm{D}$ en Ciencia Política, es Profesor de Sociología en el Centro de Pós-Grado y la Facultad de Staten Island de City University of New York, Estados Unidos. Mail de contacto: rafael.deladehesa@csi.cuny.edu 